\title{
Disentangling the Complex Vibrational Spectra of Hydrogen-bonded Clusters of 2-Pyridone with $A b$ initio Structural Search and Anharmonic Analysis
}

\author{
Jer-Lai Kuo* \\ Institute of Atomic and Molecular Sciences, Academia Sinica, Taipei 10617, Taiwan \\ E-mail: jlkuo@pub.iams.sinica.edu.tw
}

\section{Supporting Information}

An extended version of the methodology section.

Figure S1. Harmonic spectra of these global minimum structures of $2 \mathrm{PY}-\mathrm{H}_{2} \mathrm{O}, 2 \mathrm{PY}-\mathrm{MeOH}, 2 \mathrm{PY}-$ $\mathrm{Me}_{2} \mathrm{O}$, and 2PY-NH 3 predicted by MP2/aug-cc-pVDZ and comparison with experimental spectra.

Figure S2. Vibrational spectra of $2 \mathrm{PY}-\left(\mathrm{H}_{2} \mathrm{O}\right)$ measured by three research teams were reproduced here for comparison.

Figure S3. Vibrational spectra of 2PY-( $\left.\mathrm{NH}_{3}\right)$ simulated with different degrees of freedom to illustrate Fermi Resonance pattern of $\mathrm{NH}_{3}$ and the vibrational coupling between $2 \mathrm{PY}$ and $\mathrm{NH}_{3}$.

Table S1: Cartesian coordinates of three lowest energy conformers of $2 \mathrm{PY}-\mathrm{H}_{2} \mathrm{O}, 2 \mathrm{PY}-\mathrm{MeOH}, 2 \mathrm{PY}-$ $\mathrm{Me}_{2} \mathrm{O}$, and $2 \mathrm{PY}-\mathrm{NH}_{3}$ optimized by MP2/aug-cc-pVDZ.

Table S2: Cartesian coordinates of $2 \mathrm{HP}-\mathrm{H}_{2} \mathrm{O}, 2 \mathrm{HP}-\mathrm{MeOH}$, and $2 \mathrm{HP}-\mathrm{NH}_{3}$ and their corresponding transition states as optimized by MP2/aug-cc-pVDZ. 


\section{Methodology}

\section{Low-energy conformers}

Potential energy landscape of $2 \mathrm{PY}+\mathrm{X}, \mathrm{X}=\mathrm{H}_{2} \mathrm{O}, \mathrm{MeOH}, \mathrm{Me}_{2} \mathrm{O}$ and $\mathrm{NH}_{3}$ were explored using a random sampling method we developed previously. ${ }^{18-20}$ For $2 \mathrm{PY}+\mathrm{X}$, more than 100 randomly chosen initial structures first go through geometry optimization with B3LYP/6-31+G* The resulting minima were screened to identify distinct conformers for further re-optimization at MP2/aug-ccpVDZ level. Cartesian coordinates of three most stable conformers are compiled in Table S1 of ESI. All electronic structure calculations were carried out using Gaussian 16 package. ${ }^{24}$

Results from above-mentioned ab initio random structure search indicate that the structures of the global minima conformers are hydrogen-bonded with the amide group as the proton donor $-\mathrm{a}$ result in agreement with structures proposed earlier. Except $2 \mathrm{PY}-\mathrm{Me}_{2} \mathrm{O}$, all of them have two hydrogen-bonds in which the carbonyl group serves as a hydrogen-bond acceptor. Since the methylgroup of $2 \mathrm{PY}-\mathrm{MeOH}$, is not on the same plane of $2 \mathrm{PY}$, there are two conformers with the same energy. The binding energies of these global minimum structures at MP2/aug-cc-pVDZ (with zero-point correction) are roughly $10 \sim 12 \mathrm{kcal} / \mathrm{mol}$.

In these clusters, the frequency of $\mathrm{NH}$ (of 2PY) stretching mode is sensitive to the proton affinity of $\mathrm{X}$. Therefore, we can expect the frequency of $\mathrm{NH}$ of $2 \mathrm{PY}$ to red-shift from water to ammonia due to the weakening of the NH bond. Harmonic spectra of these global minimum structures predicted by MP2/aug-cc-pVDZ are shown in Figure S1 of ESI. Since the intensities are dominated by the hydrogen-bonded $\mathrm{NH}$ and $\mathrm{OH}$ stretching modes, the intensity is shown in log scale to assist comparison. From the harmonic spectra of $2 \mathrm{PY}-\left(\mathrm{H}_{2} \mathrm{O}\right)$ and $2 \mathrm{PY}-\left(\mathrm{NH}_{3}\right)$, it is clear that the oscillator strength of $\mathrm{CH}$ stretch of $2 \mathrm{PY}$ is two order of magnitude weaker than the hydrogen-bonded $\mathrm{NH}$ and $\mathrm{OH}$. 2PY-(MeOH) and 2PY-( $\left(\mathrm{Me}_{2} \mathrm{O}\right)$, it is also clear that the oscillator strength of $\mathrm{CH}$ of the methylgroups, while stronger than $\mathrm{CH}$ of $2 \mathrm{PY}$, are still at least one order weaker.

\section{$\underline{\text { Ab Initio Anharmonic Algorithms }}$}

The ab-initio anharmonic algorithm used in this work is based on discrete variable representation (DVR) ${ }^{25,26}$ approach in which the potential energy surface (PES) and dipole moment surface (DMS) are expressed on a direct product of Gauss-Hermite quadrature along the selected vibrational normal modes. Detailed descriptions on our implementations have been published elsewhere, ${ }^{21-23,27}$ we will provide a brief summary on key features used in this work.

In the literature, it has been suggested that the origin of the intensity of overtone bands can be sensitive to the choice of coordinate systems. Sibert et al. have shown that the FR coupling strength in $\mathrm{CO}_{2}$ is almost identical in rectilinear and curvilinear coordinates ${ }^{28}$ and even with coordinates of arbitrary curvature. ${ }^{29}$ For the simplicity of the kinetic energy operator in rectilinear coordinates, we use normal modes obtained by diagonalizing analytical Hessian matrix evaluated at MP2/aug-ccpVDZ at the equilibrium geometries optimized by the same level. To reduce the computational costs, the PES and DMS can be expanded using the $n$-mode representation $(n \mathrm{MR})$ scheme proposed by Bowman. ${ }^{30}$ Under $n$ MR, PES is expressed as 


$$
V\left(q_{i}, q_{j}, q_{k}, \ldots\right)=V^{(0)}+\sum_{i} \Delta \vec{V}^{(1)}\left(q_{i}\right)+\sum_{i j} \Delta V^{(2)}\left(q_{i}, q_{j}\right)+. .+\sum_{i j k} \Delta V^{(3)}\left(q_{i}, q_{j}, q_{k}\right)+. .
$$

where $V^{(0)}$ is the potential energy at the equilibrium point, $\Delta V^{(1)}$ is the change in energy within a single normal mode $q_{i}, \Delta V^{(2)}$ is the couplings between two modes $q_{i}$ and $q_{j}$, and so on. Based on the $n \mathrm{MR}$ approximation, it is possible engage more than one electronic structure methods to construct PES. ${ }^{31}$ Within nMR, PES used in this work is expressed using a mixed level scheme to balance the computational accuracy and efficiency, we choose the following settings: the $\Delta V^{(1)}$ terms are most essential and thus were calculated with CCSD/aug-cc-pVDZ level to improve accuracy of the peak position. $\Delta V^{(2)}$ and $\Delta V^{(3)}$ terms were evaluated at the MP2/aug-cc-pVDZ level. In this work, four and higher modes contributions to PES were neglected.

DMS can also been expressed in $n \mathrm{MR}$ form as

$$
\vec{u}\left(q_{i}, q_{j}, q_{k}, \ldots\right)=\vec{u}^{(0)}+\sum_{i} \Delta \vec{u}^{(1)}\left(q_{i}\right)+\sum_{i j} \Delta \vec{u}^{(2)}\left(q_{i}, q_{j}\right)+\sum_{i j k} \Delta \vec{u}^{(3)}\left(q_{i}, q_{j}, q_{k}\right)+. .
$$

To be consistent with PES, we use keep $\vec{u}^{(0)}$ and $\Delta \vec{u}^{(1)}$ at CCSD/aug-cc-pvDZ and $\Delta \vec{u}^{(2)}$ and $\Delta \vec{u}^{(3)}$ were evaluated at MP2/aug-cc-pvDZ. Four and higher modes contributions to DMS were neglected. We should note that under harmonic approximation, only the linear term of $\Delta \vec{u}^{(1)}$ play a major role in determining the oscillation strength of IR spectra.

To study Fermi resonance in the observed frequency window (above $2900 \mathrm{~cm}^{-1}$ ), we should include $\mathrm{NH} / \mathrm{OH}$ stretching modes and all (seven) vibrational modes of 2PY between 1300 to 1750 $\mathrm{cm}^{-1}$. If the multi-dimensional vibrational Hamiltonian under DVR representation is not too large to be directly diagonalized, the absorption intensities of vibrational transitions are evaluated based on the Fermi Golden rule from the eigenvectors and the DMS. To assist the assignment and analysis of the vibrational coupling behind the observed Fermi resonance pattern of 2PY, a direct product of eigenvectors of reduced dimensional Hamiltonian can be used as the basis to recast the full vibrational Hamiltonian. Such a transformation of vibrational Hamiltonian is referred to as Finite Basis Representation (FBR) ${ }^{32}$ or Pure State (PS) ${ }^{22}$ in the literature. In several previous work to analyze Fermi Resonance in solvated hydronium, ammonia, methyl-amine clusters, we have shown that the FBR representation not only function as an effective analysis tool but also offer a more efficiency way to compute IR absorption spectra.

\section{References in this section can be found in the main text.}


Figure S1. Harmonic spectra of these global minimum structures of $2 \mathrm{PY}-\mathrm{H}_{2} \mathrm{O}, 2 \mathrm{PY}-\mathrm{MeOH}, 2 \mathrm{PY}-$ $\mathrm{Me}_{2} \mathrm{O}$, and 2PY-NH${ }_{3}$ predicted by MP2/aug-cc-pVDZ and comparison with experimental spectra ${ }^{1-4}$. Please note that the scales in intensity in experimental and simulated harmonic spectra are in linear and $\log$ scales respectively. From the harmonic spectra of $2 \mathrm{PY}-\left(\mathrm{H}_{2} \mathrm{O}\right)$ and $2 \mathrm{PY}-\left(\mathrm{NH}_{3}\right)$, it is clear that the oscillator strength of $\mathrm{CH}$ stretch of 2PY (labeled in green) is two order of magnitude weaker than the hydrogen-bonded $\mathrm{NH}$ and $\mathrm{OH}$ (labeled in red). 2PY-(MeOH) and 2PY-( $\left.\mathrm{Me}_{2} \mathrm{O}\right)$, it is also clear that the oscillator strength of $\mathrm{CH}$ of the methyl-groups (labeled in blue)., while stronger than $\mathrm{CH}$ of $2 \mathrm{PY}$, are still at least one order weaker.
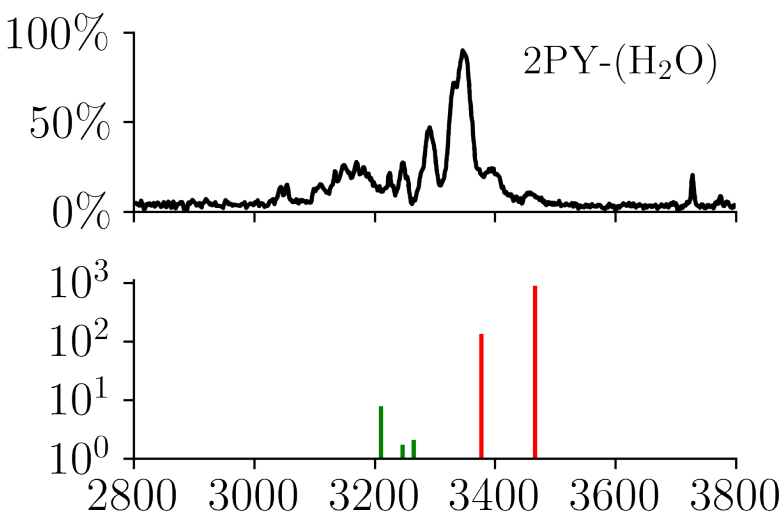

Wavenumber $\left(\mathrm{cm}^{-1}\right)$
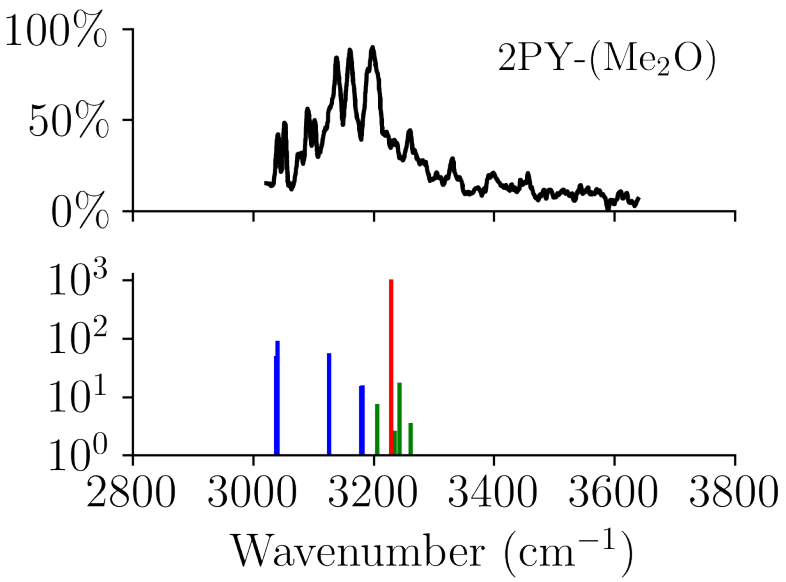
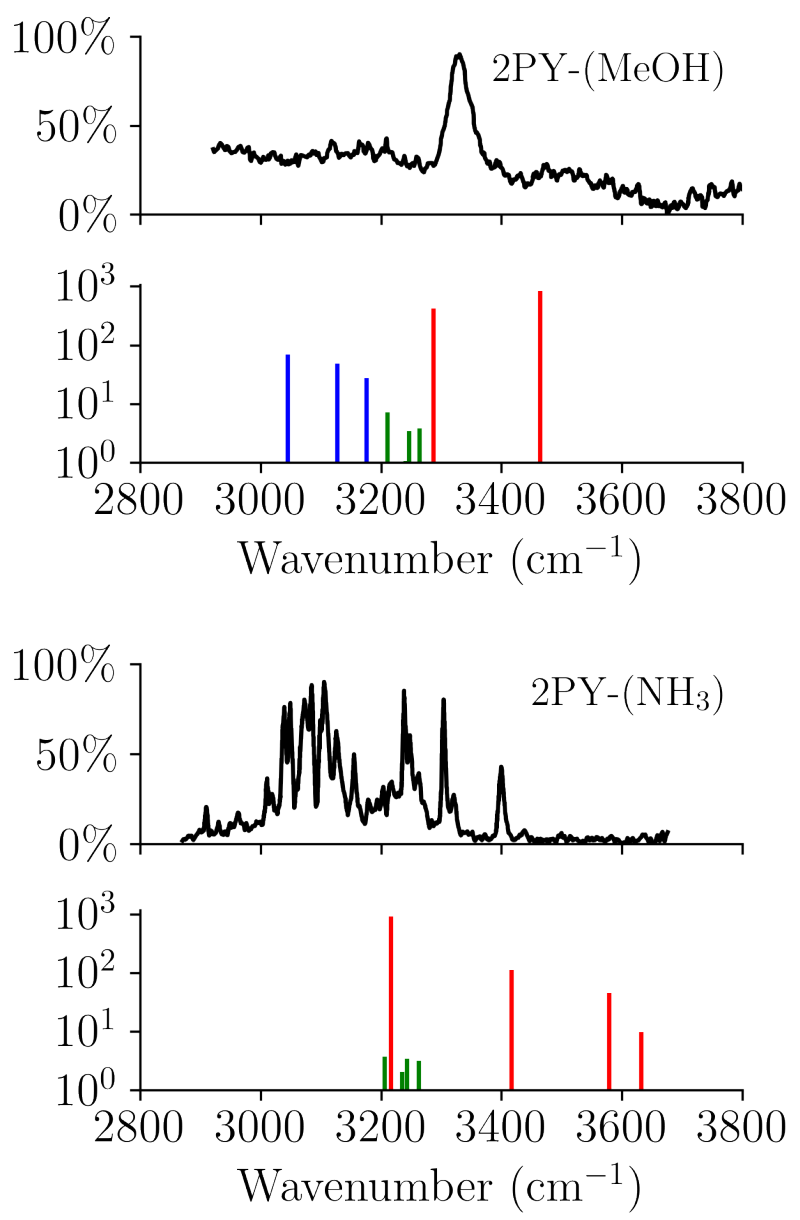
Figure S2. Vibrational spectra of 2PY- $\left(\mathrm{H}_{2} \mathrm{O}\right)$ measured by three research teams were reproduced here for comparison. (a) In the spectrum taken by Matsuda and co-workers ${ }^{1}$ the free $\mathrm{OH}$ stretch is found at $3752 \mathrm{~cm}^{-1}$ and two peaks at 3329 and $3349 \mathrm{~cm}^{-1}$ were assigned to hydrogen-bonded $\mathrm{OH}$ and $\mathrm{NH}$ stretching fundamental. Based on this assignment, the authors conclude that the structure of this cluster to form two hydrogen-bonds. (b) In the spectrum taken by Zwier and co-workers ${ }^{2}$, all three bands mentioned above were recorded along with a few minor bands between 3000 to $3300 \mathrm{~cm}^{-1}$. (c) In the spectrum taken by Leutwyler and co-workers ${ }^{4}$, the intensities of the multiple bands between 3000 to $3240 \mathrm{~cm}^{-1}$ become more pronounced. Leutwyler and co-workers concluded that this group of narrow and weak bands to arise from the $\mathrm{CH}$ stretch fundamentals of 2PY. Since weak band at 3040 $\mathrm{cm}^{-1}$ was not predicted by their rescaled harmonic calculations; they assigned this lower frequency band as an overtone or combination band.

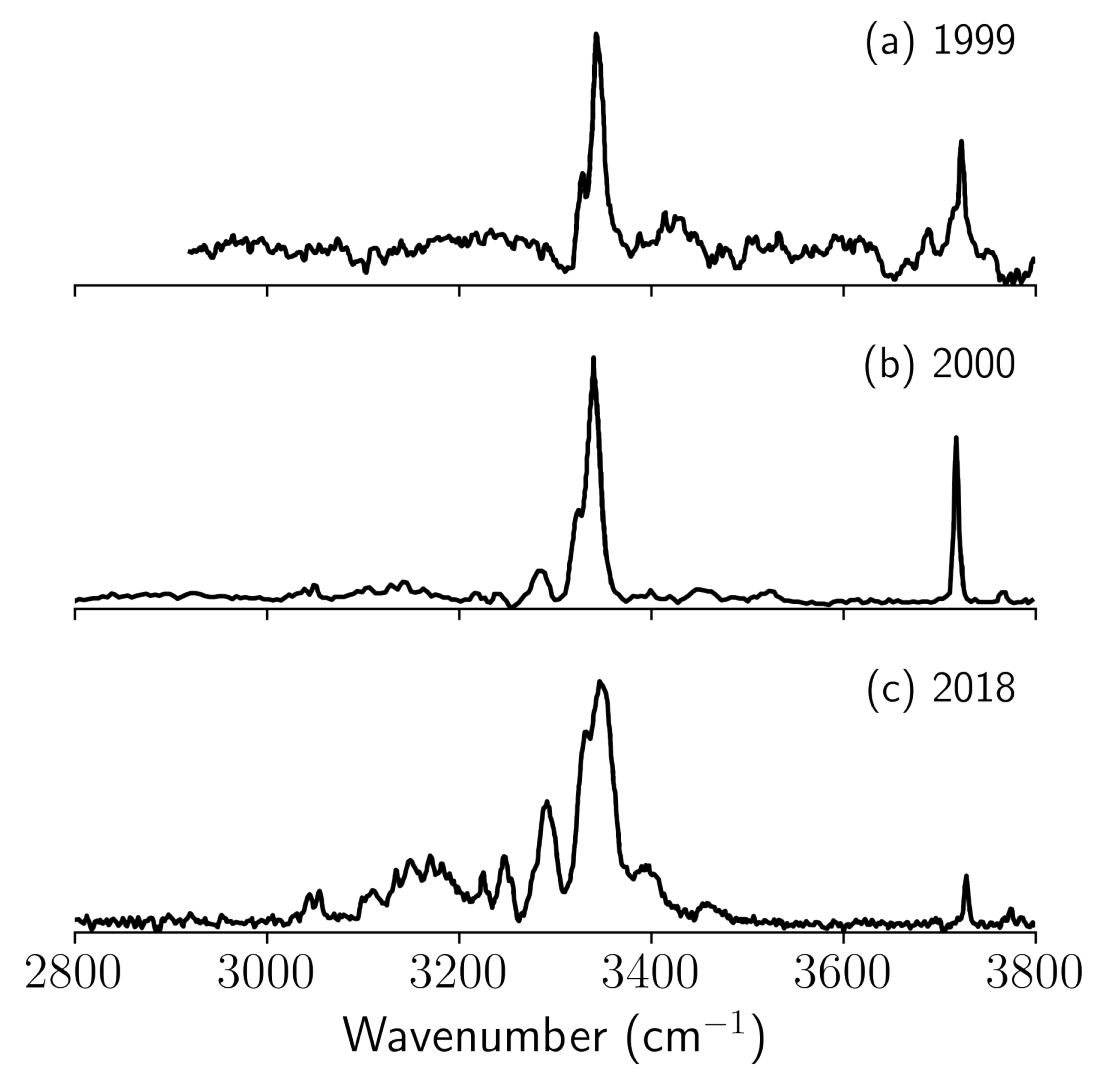

\section{References for Figure S1 and S2}

(1) Matsuda, Y.; Ebata, T.; Mikami, N. Vibrational Spectroscopy of 2-Pyridone and Its Clusters in Supersonic Jets: Structures of the Clusters as Revealed by Characteristic Shifts of the NH and $\mathrm{C}=\mathrm{O}$ Bands. J. Chem. Phys. 1999, 110 (17), 8397-8407.

(2) Florio, G. M.; Gruenloh, C. J.; Quimpo, R. C.; Zwier, T. S. The Infrared Spectroscopy of Hydrogen-Bonded Bridges: 2-Pyridone-(Water)n and 2-Hydroxypyridine-(Water) $\mathrm{n}$ Clusters, $\mathrm{N}=1,2$. J. Chem. Phys. 2000, 113 (24), 11143-11153. https://doi.org/10.1063/1.1324613.

(3) Blaser, S.; Ottiger, P.; Frey, H.-M.; Leutwyler, S. $\mathrm{NH}_{3}$ as a Strong H-Bond Donor in Singlyand Doubly-Bridged Ammonia Solvent Clusters: 2-Pyridone $\left(\mathrm{NH}_{3}\right)_{\mathrm{n}}, \mathrm{n}=1-3$. J. Phys. Chem. A 2013, 117 (32), 7523-7534. https://doi.org/10.1021/jp401786f.

(4) Siffert, L.; Blaser, S.; Ottiger, P.; Leutwyler, S. Transition from Water Wires to Bifurcated $\mathrm{H}$-Bond Networks in 2-Pyridone $\left(\mathrm{H}_{2} \mathrm{O}\right)_{\mathrm{n}}, \mathrm{n}=1-4$ Clusters. J. Phys. Chem. A 2018, 122 (48), 9285-9297. https://doi.org/10.1021/acs.jpca.8b09410. 
Figure S3. Vibrational spectra of 2PY-( $\left.\mathrm{NH}_{3}\right)$ simulated by (a) using $\mathrm{NH}$ stretching of 2PY only, (b) six-dimensional calculation using NH stretch of $2 \mathrm{PY}$ and 3 stretch and 2 bending modes of $\mathrm{NH}_{3}$. FR pattern of NH3 are marked with red asterisks. Comparing two spectra, it is clear that the vibrational coupling between $\mathrm{NH}$ in $2 \mathrm{PY}$ and $\mathrm{NH}_{3}$ is not strong only induce a slight red-shift by $\mathrm{NH}$ stretching fundamental by less than $10 \mathrm{~cm}^{-1}$.

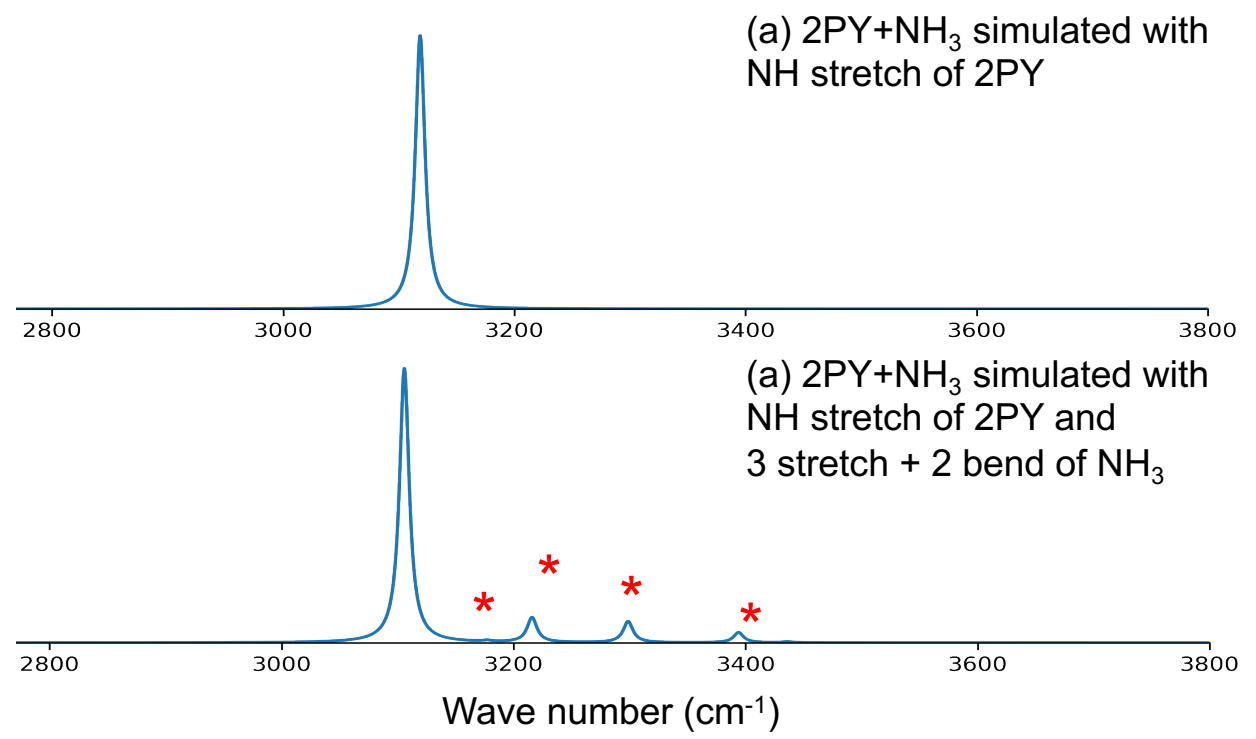


Table S1. Cartesian coordinates of three most stable conformers $2 \mathrm{PY}-\mathrm{X}, \mathrm{X}=\mathrm{H}_{2} \mathrm{O}, \mathrm{MeOH}, \mathrm{Me}_{2} \mathrm{O}$ and $\mathrm{NH}_{3}$ optimized at the MP2/aug-cc-pVDZ level.

\begin{tabular}{|c|c|c|c|}
\hline \multicolumn{4}{|c|}{ 2PY-( $\left(\mathrm{H}_{2} \mathrm{O}\right) \_\mathrm{a}$} \\
\hline C & -2.10356 & -0.94048 & -0.00097 \\
\hline C & -0.83035 & -1.47309 & 0.00729 \\
\hline C & 0.22174 & 0.74688 & 0.00624 \\
\hline C & -1.11741 & 1.29758 & 0.00005 \\
\hline C & -2.23467 & 0.48028 & -0.00398 \\
\hline $\mathrm{H}$ & -2.97171 & -1.60009 & -0.00544 \\
\hline H & -0.62533 & -2.54538 & 0.00995 \\
\hline 0 & 1.28511 & 1.41419 & 0.00603 \\
\hline $\mathrm{H}$ & -1.20158 & 2.38636 & -0.00320 \\
\hline H & -3.23145 & 0.93222 & -0.01005 \\
\hline N & 0.26261 & -0.65240 & 0.01176 \\
\hline $\mathrm{H}$ & 1.21206 & -1.04958 & 0.02322 \\
\hline 0 & 3.07315 & -0.64299 & -0.08843 \\
\hline $\mathrm{H}$ & 2.69818 & 0.26775 & -0.03434 \\
\hline $\mathrm{H}$ & 3.80097 & -0.66104 & 0.54495 \\
\hline \multicolumn{4}{|c|}{ 2PY-( $\left(\mathrm{H}_{2} \mathrm{O}\right) \_b$} \\
\hline C & -2.26164 & 0.79657 & -0.00002 \\
\hline C & -2.20797 & -0.58066 & -0.00001 \\
\hline C & 0.26011 & -0.58689 & -0.00007 \\
\hline C & 0.18954 & 0.86227 & 0.00005 \\
\hline C & -1.02856 & 1.51867 & 0.00003 \\
\hline $\mathrm{H}$ & -3.22717 & 1.30317 & -0.00003 \\
\hline $\mathrm{H}$ & -3.09008 & -1.22335 & -0.00002 \\
\hline 0 & 1.29669 & -1.28745 & 0.00010 \\
\hline H & 1.13800 & 1.40442 & 0.00011 \\
\hline H & -1.04457 & 2.61283 & 0.00005 \\
\hline $\mathrm{N}$ & -0.99229 & -1.21460 & -0.00004 \\
\hline $\mathrm{H}$ & -0.94923 & -2.23200 & -0.00004 \\
\hline 0 & 3.37110 & 0.60170 & -0.00008 \\
\hline $\mathrm{H}$ & 4.27120 & 0.25548 & 0.00006 \\
\hline $\mathrm{H}$ & 2.79658 & -0.19212 & 0.00006 \\
\hline \multicolumn{4}{|c|}{$2 \mathrm{PY}-\left(\mathrm{H}_{2} \mathrm{O}\right)_{-} \mathrm{c}$} \\
\hline C & 0.92853 & 0.26927 & 0.00004 \\
\hline C & 0.36089 & -0.98676 & 0.00007 \\
\hline C & -1.94427 & -0.07936 & -0.00027 \\
\hline C & -1.32627 & 1.23744 & -0.00002 \\
\hline C & 0.04744 & 1.39612 & 0.00003 \\
\hline $\mathrm{H}$ & 2.01448 & 0.36946 & 0.00011 \\
\hline $\mathrm{H}$ & 0.94419 & -1.90977 & 0.00017 \\
\hline 0 & -3.16116 & -0.33258 & 0.00011 \\
\hline $\mathrm{H}$ & -2.00350 & 2.09456 & 0.00007 \\
\hline $\mathrm{H}$ & 0.46853 & 2.40676 & 0.00011 \\
\hline $\mathrm{N}$ & -1.00363 & -1.12773 & -0.00003 \\
\hline $\mathrm{H}$ & -1.41924 & -2.05698 & 0.00004 \\
\hline 0 & 4.27271 & -0.14732 & -0.00003 \\
\hline $\mathrm{H}$ & 4.86537 & -0.09554 & -0.76126 \\
\hline $\mathrm{H}$ & 4.86531 & -0.09540 & 0.76123 \\
\hline
\end{tabular}




\begin{tabular}{lrrr}
\multicolumn{2}{l}{ PY-(NH$) \_a$} \\
$C$ & 2.02893 & -1.07987 & 0.00021 \\
$\mathrm{C}$ & 0.70850 & -1.48363 & -0.00070 \\
$\mathrm{C}$ & -0.12546 & 0.82860 & -0.00048 \\
$\mathrm{C}$ & 1.26520 & 1.24419 & 0.00049 \\
$\mathrm{C}$ & 2.29756 & 0.32212 & 0.00083 \\
$\mathrm{H}$ & 2.82862 & -1.82117 & 0.00042 \\
$\mathrm{H}$ & 0.40132 & -2.53188 & -0.00128 \\
$\mathrm{O}$ & -1.11821 & 1.59091 & -0.00081 \\
$\mathrm{H}$ & 1.45699 & 2.31954 & 0.00093 \\
$\mathrm{H}$ & 3.33388 & 0.67495 & 0.00155 \\
$\mathrm{~N}$ & -0.30052 & -0.56348 & -0.00097 \\
$\mathrm{H}$ & -1.29243 & -0.86881 & -0.00178 \\
$\mathrm{~N}$ & -3.18144 & -0.62275 & 0.00064 \\
$\mathrm{H}$ & -3.76593 & -0.77566 & 0.82223 \\
$\mathrm{H}$ & -3.77609 & -0.77830 & -0.81309 \\
$\mathrm{H}$ & -2.91534 & 0.36923 & -0.00221
\end{tabular}

2PY- $\left(\mathrm{NH}_{3}\right) \_b$

$\begin{array}{lrrr}\text { C } & -2.23909 & 0.90779 & 0.00006 \\ \text { C } & -2.28327 & -0.46963 & 0.00014 \\ \text { C } & 0.18277 & -0.65310 & -0.00011 \\ \text { C } & 0.21200 & 0.79981 & -0.00017 \\ \text { C } & -0.95660 & 1.54024 & -0.00009 \\ \text { H } & -3.16632 & 1.48162 & 0.00012 \\ \text { H } & -3.20969 & -1.04694 & 0.00026 \\ \text { O } & 1.16638 & -1.42110 & -0.00013 \\ \text { H } & 1.20036 & 1.26768 & -0.00029 \\ \text { H } & -0.89498 & 2.63298 & -0.00015 \\ \text { N } & -1.11618 & -1.18938 & 0.00005 \\ \text { H } & -1.14638 & -2.20710 & 0.00010 \\ \text { N } & 3.55717 & 0.57975 & 0.00008 \\ \text { H } & 2.96914 & -0.26048 & -0.00066 \\ \text { H } & 4.16648 & 0.50887 & 0.81544 \\ \text { H } & 4.16858 & 0.50897 & -0.81371\end{array}$

2PY- $\left(\mathrm{NH}_{3}\right)_{-} \mathrm{c}$

$\begin{array}{lrrr}\text { C } & 0.31052 & 1.80313 & 0.00092 \\ \text { C } & 1.63740 & 1.43108 & -0.00143 \\ \text { C } & 1.07564 & -0.98203 & 0.00027 \\ \text { C } & -0.31629 & -0.56129 & 0.00280 \\ \text { C } & -0.68155 & 0.77188 & 0.00308 \\ \text { H } & 0.04314 & 2.86030 & 0.00118 \\ \text { H } & 2.46598 & 2.14212 & -0.00315 \\ \text { O } & 1.50529 & -2.14828 & -0.00026 \\ \text { H } & -1.06954 & -1.35217 & 0.00461 \\ \text { H } & -1.74832 & 1.01516 & 0.00492 \\ \text { N } & 1.97641 & 0.10208 & -0.00172 \\ \text { H } & 2.95656 & -0.17260 & -0.00348 \\ \text { N } & -3.97320 & -0.20846 & -0.00102 \\ \text { H } & -3.99728 & -0.81794 & -0.81937 \\ \text { H } & -4.85483 & 0.30495 & -0.00802 \\ \text { H } & -4.01480 & -0.82555 & 0.81089\end{array}$

2PY-(MeOH)_a 


\begin{tabular}{|c|c|c|c|}
\hline C & -2.43998 & -1.09937 & 0.09144 \\
\hline C & -1.13477 & -1.48727 & -0.13686 \\
\hline C & -0.32000 & 0.82867 & -0.09434 \\
\hline C & -1.68998 & 1.22825 & 0.14782 \\
\hline C & -2.70848 & 0.29424 & 0.23607 \\
\hline $\mathrm{H}$ & -3.22948 & -1.84868 & 0.15596 \\
\hline $\mathrm{H}$ & -0.82829 & -2.52772 & -0.26197 \\
\hline 0 & 0.66250 & 1.60598 & -0.19334 \\
\hline $\mathrm{H}$ & -1.88042 & 2.29827 & 0.25438 \\
\hline $\mathrm{H}$ & -3.73339 & 0.63232 & 0.41816 \\
\hline $\mathrm{N}$ & -0.14087 & -0.55347 & -0.21990 \\
\hline $\mathrm{H}$ & 0.84113 & -0.83276 & -0.38555 \\
\hline 0 & 2.60922 & -0.27594 & -0.56964 \\
\hline $\mathrm{H}$ & 2.14233 & 0.59102 & -0.51954 \\
\hline C & 3.40214 & -0.39222 & 0.62148 \\
\hline $\mathrm{H}$ & 3.85212 & -1.39421 & 0.61732 \\
\hline $\mathrm{H}$ & 2.78617 & -0.27692 & 1.53062 \\
\hline $\mathrm{H}$ & 4.20842 & 0.35878 & 0.64014 \\
\hline \multicolumn{4}{|c|}{ 2PY-(MeOH)_a' } \\
\hline C & $\overline{2} .43969$ & -1.09943 & 0.09166 \\
\hline C & 1.13460 & -1.48719 & -0.13761 \\
\hline C & 0.31992 & 0.82881 & -0.09485 \\
\hline C & 1.68973 & 1.22820 & 0.14839 \\
\hline C & 2.70813 & 0.29410 & 0.23697 \\
\hline $\mathrm{H}$ & 3.22910 & -1.84881 & 0.15643 \\
\hline $\mathrm{H}$ & 0.82818 & -2.52759 & -0.26326 \\
\hline 0 & -0.66254 & 1.60613 & -0.19420 \\
\hline $\mathrm{H}$ & 1.88024 & 2.29815 & 0.25552 \\
\hline $\mathrm{H}$ & 3.73291 & 0.63213 & 0.41987 \\
\hline $\mathrm{N}$ & 0.14083 & -0.55333 & -0.22104 \\
\hline $\mathrm{H}$ & -0.84112 & -0.83251 & -0.38707 \\
\hline 0 & -2.60949 & -0.27568 & -0.56946 \\
\hline $\mathrm{H}$ & -2.14259 & 0.59127 & -0.51943 \\
\hline C & -3.40107 & -0.39263 & 0.62255 \\
\hline $\mathrm{H}$ & -3.85091 & -1.39468 & 0.61837 \\
\hline $\mathrm{H}$ & -4.20738 & 0.35828 & 0.64243 \\
\hline $\mathrm{H}$ & -2.78404 & -0.27770 & 1.53100 \\
\hline \multicolumn{4}{|c|}{ 2PY-(MeOH) b } \\
\hline C & 2.38917 & -1.19241 & 0.00086 \\
\hline C & 1.03699 & -1.46859 & 0.00032 \\
\hline C & 0.42344 & 0.91497 & -0.00063 \\
\hline C & 1.84983 & 1.19359 & -0.0000 \\
\hline C & 2.78987 & 0.17854 & 0.00068 \\
\hline $\mathrm{H}$ & 3.11426 & -2.00676 & 0.0013 \\
\hline $\mathrm{H}$ & 0.63068 & -2.48236 & 0.00035 \\
\hline 0 & -0.49022 & 1.76426 & -0.00136 \\
\hline $\mathrm{H}$ & 2.14289 & 2.24583 & -0.00022 \\
\hline $\mathrm{H}$ & 3.85500 & 0.43139 & 0.00109 \\
\hline $\mathrm{N}$ & 0.11925 & -0.45583 & -0.00035 \\
\hline $\mathrm{H}$ & -0.88288 & -0.68890 & -0.00078 \\
\hline 0 & -2.71322 & -0.97554 & -0.00398 \\
\hline $\mathrm{H}$ & -3.33527 & -1.71361 & 0.00295 \\
\hline C & -3.46247 & 0.26523 & 0.00314 \\
\hline $\mathrm{H}$ & -2.71203 & 1.06547 & -0.00204 \\
\hline
\end{tabular}




$\begin{array}{rrrr}H & -4.08318 & 0.34035 & 0.90941 \\ H & -4.09768 & 0.34166 & -0.89291\end{array}$

$\begin{array}{lrrr}\text { 2PY-(MeOH) } \mathrm{C} & & \\ \mathrm{C} & -2.82228 & 0.81952 & -0.00030 \\ \mathrm{C} & -2.78687 & -0.55829 & -0.00060 \\ \mathrm{C} & -0.31978 & -0.59668 & 0.00048 \\ \mathrm{C} & -0.37040 & 0.85326 & 0.00069 \\ \mathrm{C} & -1.57993 & 1.52548 & 0.00034 \\ \mathrm{H} & -3.78121 & 1.33854 & -0.00059 \\ \mathrm{H} & -3.67721 & -1.18943 & -0.00112 \\ \text { O } & 0.70830 & -1.31088 & 0.00079 \\ \mathrm{H} & 0.58586 & 1.38213 & 0.00112 \\ \mathrm{H} & -1.58177 & 2.61973 & 0.00054 \\ \mathrm{~N} & -1.57945 & -1.20814 & -0.00024 \\ \mathrm{H} & -1.54978 & -2.22603 & -0.00042 \\ \mathrm{O} & 2.78010 & 0.56295 & -0.00084 \\ \mathrm{H} & 2.18320 & -0.21342 & -0.00025 \\ \mathrm{C} & 4.12694 & 0.08821 & -0.00015 \\ \mathrm{H} & 4.78327 & 0.96962 & -0.00232 \\ \mathrm{H} & 4.35004 & -0.51689 & -0.89652 \\ \mathrm{H} & 4.35054 & -0.51286 & 0.89881\end{array}$

$\begin{array}{lrrr}2 \mathrm{PY}-\left(\mathrm{Me}_{2} \mathrm{O}\right) \mathrm{a} & & \\ \mathrm{C} & -1.29818 & -2.59934 & 0.00000 \\ \mathrm{C} & -1.51605 & -1.23616 & 0.00000 \\ \mathrm{C} & 0.89104 & -0.72839 & 0.00000 \\ \mathrm{C} & 1.10979 & -2.16421 & 0.00000 \\ \mathrm{C} & 0.05398 & -3.05900 & 0.00000 \\ \mathrm{H} & -2.14260 & -3.28926 & 0.00000 \\ \mathrm{H} & -2.51102 & -0.78523 & 0.00000 \\ \mathrm{O} & 1.77769 & 0.15202 & 0.00000 \\ \mathrm{H} & 2.14822 & -2.50303 & 0.00000 \\ \mathrm{H} & 0.25995 & -4.13420 & 0.00000 \\ \mathrm{~N} & -0.46397 & -0.36387 & 0.00000 \\ \mathrm{H} & -0.64251 & 0.65763 & 0.00000 \\ \mathrm{O} & -0.69387 & 2.43694 & 0.00000 \\ \mathrm{C} & 0.05398 & 2.78333 & 1.17980 \\ \mathrm{C} & 0.05398 & 2.78333 & -1.17980 \\ \mathrm{H} & -0.56234 & 2.49634 & 2.04243 \\ \mathrm{H} & 0.23650 & 3.87158 & 1.20628 \\ \mathrm{H} & 1.01281 & 2.23812 & 1.19617 \\ \mathrm{H} & -0.56234 & 2.49634 & -2.04243 \\ \mathrm{H} & 1.01281 & 2.23812 & -1.19617 \\ \mathrm{H} & 0.23650 & 3.87158 & -1.20628 \\ \text { 2PY-(Me } 2 \mathrm{O}) \mathrm{b} & & & \\ \mathrm{C} & 2.50621 & -1.47724 & 0.01961 \\ \mathrm{C} & 1.12649 & -1.46963 & -0.01851 \\ \mathrm{C} & 1.01447 & 0.98856 & -0.01750 \\ \mathrm{C} & 2.46726 & 0.96877 & 0.02295 \\ \mathrm{C} & 3.17909 & -0.21754 & 0.04065 \\ \mathrm{H} & 3.04879 & -2.42296 & 0.03242 \\ \mathrm{H} & 0.52167 & -2.37903 & -0.03825 \\ \mathrm{O} & 0.29458 & 2.00720 & -0.03616\end{array}$




\begin{tabular}{|c|c|c|c|}
\hline $\mathrm{H}$ & 2.96990 & 1.93843 & 0.03824 \\
\hline $\mathrm{H}$ & 4.27305 & -0.18839 & 0.07079 \\
\hline $\mathrm{N}$ & 0.43554 & -0.29074 & -0.03522 \\
\hline $\mathrm{H}$ & -0.59523 & -0.31369 & -0.0652 \\
\hline 0 & -2.41357 & -0.26283 & -0.1675 \\
\hline C & -2.89836 & 1.06826 & 0.0779 \\
\hline C & -3.43107 & -1.23828 & 0.0563 \\
\hline $\mathrm{H}$ & -2.04304 & 1.74274 & -0.0567 \\
\hline $\mathrm{H}$ & -3.28456 & 1.14913 & 1.10969 \\
\hline $\mathrm{H}$ & -3.70585 & 1.31302 & -0.63415 \\
\hline $\mathrm{H}$ & -2.99693 & -2.22242 & -0.1658 \\
\hline $\mathrm{H}$ & -4.29410 & -1.06017 & -0.6097 \\
\hline $\mathrm{H}$ & -3.77496 & -1.21378 & 1.10621 \\
\hline \multicolumn{4}{|c|}{ 2PY-( $\left(\mathrm{Me}_{2} \mathrm{O}\right) \_\mathrm{c}$} \\
\hline C & 2.78900 & -1.42040 & 0.0000 \\
\hline C & 3.30622 & -0.14313 & 0.0006 \\
\hline C & 1.05280 & 0.88294 & -0.0000 \\
\hline C & 0.52472 & -0.47185 & -0.0001 \\
\hline C & 1.36656 & -1.56967 & -0.0006 \\
\hline $\mathrm{H}$ & 3.46063 & -2.27946 & 0.00002 \\
\hline $\mathrm{H}$ & 4.37520 & 0.07815 & 0.0001 \\
\hline 0 & 0.39637 & 1.94132 & -0.0001 \\
\hline $\mathrm{H}$ & -0.56280 & -0.58554 & -0.0001 \\
\hline $\mathrm{H}$ & 0.93054 & -2.57362 & -0.0001 \\
\hline $\mathrm{N}$ & 2.45972 & 0.93531 & 0.0000 \\
\hline $\mathrm{H}$ & 2.83971 & 1.87980 & 0.0000 \\
\hline 0 & -2.75946 & -0.37469 & 0.0008 \\
\hline C & -4.02425 & -1.02803 & -0.0003 \\
\hline C & -2.92085 & 1.04816 & -0.0000 \\
\hline $\mathrm{H}$ & -3.83463 & -2.10992 & 0.0004 \\
\hline $\mathrm{H}$ & -4.60898 & -0.75899 & -0.9002 \\
\hline $\mathrm{H}$ & -4.61109 & -0.75804 & 0.89789 \\
\hline $\mathrm{H}$ & -1.91613 & 1.49112 & 0.0007 \\
\hline $\mathrm{H}$ & -3.47637 & 1.37453 & 0.89880 \\
\hline $\mathrm{H}$ & -3.47460 & 1.37370 & -0.90021 \\
\hline
\end{tabular}


Table S2: Cartesian coordinates of $2 \mathrm{HP}-\mathrm{H}_{2} \mathrm{O}, 2 \mathrm{HP}-\mathrm{MeOH}$, and $2 \mathrm{HP}-\mathrm{NH}_{3}$ and their corresponding transition states as optimized by MP2/aug-cc-pVDZ.

$\begin{array}{lrrr}\left.\text { 2HP-(H } \mathrm{H}_{2} \mathrm{O}\right) \text { min } & & \\ \mathrm{C} & -2.09497 & -0.94678 & 0.00260 \\ \mathrm{C} & -0.79495 & -1.46188 & -0.00745 \\ \mathrm{C} & 0.14795 & 0.65272 & -0.00149 \\ \mathrm{C} & -1.12273 & 1.27042 & 0.00923 \\ \mathrm{C} & -2.25645 & 0.45416 & 0.01136 \\ \mathrm{H} & -2.95409 & -1.62121 & 0.00251 \\ \mathrm{H} & -0.61792 & -2.54157 & -0.01601 \\ \mathrm{O} & 1.24060 & 1.45375 & -0.00498 \\ \mathrm{H} & -1.18693 & 2.36063 & 0.01424 \\ \mathrm{H} & -3.25442 & 0.90172 & 0.01883 \\ \mathrm{~N} & 0.31622 & -0.68297 & -0.00867 \\ \mathrm{H} & 2.20951 & -1.01499 & -0.01976 \\ \mathrm{O} & 3.09666 & -0.59342 & -0.08408 \\ \mathrm{H} & 2.04489 & 0.88174 & -0.01741 \\ \mathrm{H} & 3.57425 & -0.88001 & 0.70530\end{array}$

2HP- $\left(\mathrm{H}_{2} \mathrm{O}\right) \_\mathrm{TS}$

$\begin{array}{lrrr}\text { C } & -2.07085 & -0.86711 & 0.00409 \\ \mathrm{C} & -0.80711 & -1.44793 & -0.00799 \\ \mathrm{C} & 0.27188 & 0.67951 & -0.00369 \\ \mathrm{C} & -0.99943 & 1.32115 & 0.01036 \\ \mathrm{C} & -2.15694 & 0.54677 & 0.01356 \\ \mathrm{H} & -2.96314 & -1.49543 & 0.00463 \\ \mathrm{H} & -0.65714 & -2.53033 & -0.01786 \\ \mathrm{O} & 1.40369 & 1.33474 & -0.01107 \\ \mathrm{H} & -1.03011 & 2.41257 & 0.01607 \\ \mathrm{H} & -3.13535 & 1.03632 & 0.02278 \\ \mathrm{~N} & 0.31751 & -0.68967 & -0.01105 \\ \mathrm{H} & 1.51085 & -0.99726 & -0.00598 \\ \mathrm{O} & 2.73962 & -0.66129 & -0.08173 \\ \mathrm{H} & 2.24239 & 0.48168 & -0.02133 \\ \mathrm{H} & 3.23813 & -0.86184 & 0.72347\end{array}$

2HP-(MeOH)_min

$\begin{array}{lrrr}\text { C } & -2.43346 & -1.08190 & 0.08938 \\ \text { C } & -1.11600 & -1.46831 & -0.17607 \\ \text { C } & -0.35650 & 0.71927 & -0.10501 \\ \text { C } & -1.65368 & 1.20921 & 0.16717 \\ \text { C } & -2.70243 & 0.29141 & 0.26466 \\ \text { H } & -3.22432 & -1.83228 & 0.15623 \\ \text { H } & -0.85695 & -2.52144 & -0.32107 \\ \text { O } & 0.65498 & 1.61444 & -0.19907 \\ \text { H } & -1.80376 & 2.28376 & 0.29165 \\ \text { H } & -3.71805 & 0.63969 & 0.47340 \\ \text { N } & -0.08632 & -0.58982 & -0.27357 \\ \text { H } & 1.80171 & -0.70924 & -0.58029 \\ \text { O } & 2.64274 & -0.19813 & -0.58208 \\ \text { H } & 1.49029 & 1.11378 & -0.39192 \\ \text { C } & 3.28539 & -0.44822 & 0.68027 \\ \text { H } & 3.64064 & -1.48914 & 0.74598 \\ \text { H } & 2.60465 & -0.24287 & 1.52442\end{array}$


2HP-(MeOH)_TS

$\begin{array}{lr}\mathrm{C} & -2.35752 \\ \mathrm{C} & -1.04787 \\ \mathrm{C} & -0.24143 \\ \mathrm{C} & -1.56765 \\ \mathrm{C} & -2.61086 \\ \mathrm{H} & -3.15732 \\ \mathrm{H} & -0.77600 \\ \mathrm{O} & 0.78679 \\ \mathrm{H} & -1.72913 \\ \mathrm{H} & -3.62882 \\ \mathrm{~N} & -0.03159 \\ \mathrm{H} & 1.22873 \\ \mathrm{O} & 2.34410 \\ \mathrm{H} & 1.75421 \\ \mathrm{C} & 3.09321 \\ \mathrm{H} & 3.51592 \\ \mathrm{H} & 2.44645 \\ \mathrm{H} & 3.91274\end{array}$

$\begin{array}{rr}-1.06344 & 0.07932 \\ -1.46548 & -0.16340 \\ 0.77872 & -0.09274 \\ 1.23925 & 0.15910 \\ 0.32073 & 0.24298 \\ -1.80377 & 0.13864 \\ -2.51528 & -0.30234 \\ 1.57773 & -0.18654 \\ 2.31267 & 0.27821 \\ 0.67343 & 0.43467 \\ -0.57016 & -0.24458 \\ -0.69557 & -0.44889 \\ -0.21338 & -0.56312 \\ 0.80098 & -0.41017 \\ -0.48275 & 0.63823 \\ -1.49613 & 0.57979 \\ -0.40695 & 1.53090 \\ 0.24475 & 0.72761\end{array}$

2HP-( $\left.\mathrm{NH}_{3}\right) \_$min

\begin{tabular}{|c|c|c|c|}
\hline C & -2.18799 & -0.90449 & 0.00013 \\
\hline C & -0.90540 & -1.46445 & -0.00018 \\
\hline C & 0.11618 & 0.60851 & -0.00020 \\
\hline C & -1.13320 & 1.27255 & 0.00011 \\
\hline C & -2.29807 & 0.50129 & 0.00028 \\
\hline $\mathrm{H}$ & -3.07095 & -1.54781 & 0.00023 \\
\hline $\mathrm{H}$ & -0.77266 & -2.55126 & -0.00030 \\
\hline 0 & 1.23294 & 1.37058 & -0.00036 \\
\hline $\mathrm{H}$ & -1.15671 & 2.36466 & 0.00019 \\
\hline $\mathrm{H}$ & -3.27857 & 0.98652 & 0.00052 \\
\hline $\mathrm{N}$ & 0.23779 & -0.73344 & -0.00033 \\
\hline $\mathrm{H}$ & 2.52736 & -1.18987 & -0.00286 \\
\hline $\mathrm{N}$ & 3.27520 & -0.49057 & 0.00013 \\
\hline $\mathrm{H}$ & 2.03410 & 0.77244 & -0.00067 \\
\hline $\mathrm{H}$ & 3.85281 & -0.65541 & 0.82372 \\
\hline $\mathrm{H}$ & 3.86106 & -0.65633 & -0.81739 \\
\hline \multicolumn{4}{|c|}{ 2HP-(NH$\left.)_{3}\right)_{T S}$} \\
\hline C & 2.08114 & -0.93035 & 0.00003 \\
\hline C & 0.79041 & -1.45196 & -0.00010 \\
\hline C & -0.22638 & 0.71000 & -0.00009 \\
\hline C & 1.08923 & 1.28937 & 0.00007 \\
\hline C & 2.22046 & 0.48029 & 0.00013 \\
\hline $\mathrm{H}$ & 2.94650 & -1.59626 & 0.00006 \\
\hline $\mathrm{H}$ & 0.61749 & -2.53375 & -0.00018 \\
\hline 0 & -1.30021 & 1.42831 & -0.00016 \\
\hline $\mathrm{H}$ & 1.16314 & 2.37980 & 0.00015 \\
\hline $\mathrm{H}$ & 3.21593 & 0.93635 & 0.00025 \\
\hline $\mathrm{N}$ & -0.32256 & -0.67340 & -0.00017 \\
\hline $\mathrm{H}$ & -1.74572 & -0.97463 & 0.00023 \\
\hline $\mathrm{N}$ & -2.85473 & -0.60690 & 0.00018 \\
\hline $\mathrm{H}$ & -3.38657 & -0.84208 & 0.83634 \\
\hline
\end{tabular}




$\begin{array}{rrrr}H & -3.38629 & -0.84184 & -0.83622 \\ H & -2.51091 & 0.42394 & 0.00024\end{array}$

